\title{
Multinucleated Giant Hemocytes Are Effector Cells in Cell-Mediated Immune Responses of Drosophila
}

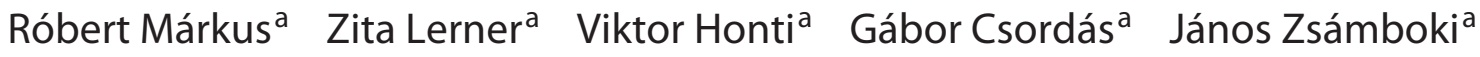 \\ Gyöngyi Cinege $^{a}$ Árpád Párducz ${ }^{b}$ Tamás Lukacsovich ${ }^{c}$ Éva Kurucz ${ }^{a}$ \\ István Andóa \\ ${ }^{a}$ Immunology Unit, Institute of Genetics, and b Laboratory of Molecular Neurobiology, Institute of Biophysics, \\ Biological Research Centre, Hungarian Academy of Sciences, Szeged, Hungary; ${ }^{\mathrm{C} D e p a r t m e n t}$ of Molecular, \\ Cell and Developmental Biology, University of California, Irvine, Calif., USA
}

\section{Key Words \\ Comparative immunology - Drosophila - Encapsulation · Granuloma - Host defense - Immune responses - Insects . Multinucleated giant hemocytes · Parasitology}

\begin{abstract}
We identified and characterized a so far unrecognized cell type, dubbed the multinucleated giant hemocyte (MGH), in the ananassae subgroup of Drosophilidae. Here, we describe the functional and ultrastructural characteristics of this novel blood cell type as well as its characterization with a set of discriminative immunological markers. MGHs are encapsulating cells that isolate and kill the parasite without melanization. They share some properties with but differ considerably from lamellocytes, the encapsulating cells of Drosophila melanogaster, the broadly used model organism in studies of innate immunity. MGHs are nonproliferative effector cells that are derived from phagocytic cells of the sessile tissue and the circulation, but do not exhibit phagocytic activity. In contrast to lamellocytes, MGHs are gigantic cells with filamentous projections and contain many nuclei, which are the result of the fusion of several cells. Although the structure of lamellocytes and MGHs differ remarkably, their function in
\end{abstract}

the elimination of parasites is similar, which is potentially the result of the convergent evolution of interactions between hosts and parasites in different geographic regions. MGHs are highly motile and share several features with mammalian multinucleated giant cells, a syncytium of macrophages formed during granulomatous inflammation.

두 2015 S. Karger AG, Base

\section{Introduction}

Granuloma formation and the encapsulation reaction are typical responses in the animal kingdom to chronic inflammatory stimuli [1]. In fact, the capsule formation of Drosophila melanogaster larvae in response to parasitoid infection serves as a viable model for foreign-bodytype granulomas [2-5]. In D. melanogaster, blood cells, referred to as hemocytes, are responsible for the elimination of microorganisms and multicellular parasites [6-8]. Based on their functions, three main classes of hemocytes were identified: plasmatocytes, lamellocytes and crystal

R.M., Z.L. and V.H. contributed equally to this work.

\section{KARGER 125}

(c) 2015 S. Karger AG, Base

$1662-811 X / 15 / 0074-0340 \$ 39.50 / 0$

E-Mail karger@karger.com

www.karger.com/jin
Dr. István Andó or Dr. Éva Kurucz

Immunology Unit, Institute of Genetics, Biological Research Centre

Hungarian Academy of Sciences

HU-6701 Szeged (Hungary)

E-Mail ando@brc.hu or kurucz@brc.hu 
cells. Plasmatocytes, the major hemocyte type in the circulation, are spherical, adhesive cells that engulf microbes and cell debris [9]. In response to immune induction by tumors or eggs of parasitoid wasps, they differentiate into lamellocytes $[9,10]$. Lamellocytes are large, flattened cells that are not phagocytic, but isolate larger objects, such as tumors and eggs of multicellular parasites (e.g. eggs of parasitoid wasps) by forming multilayered melanizing capsules around them [7]. Crystal cells contain crystallized prophenol oxidases, the key components of the melanization reaction $[6,11]$.

Hemocytes of D. melanogaster larvae reside in three hematopoietic compartments $[3,12]$ : the lymph gland, the sessile hematopoietic tissue and the circulation. The lymph gland is a compact hematopoietic organ formed by hemocytes in various stages of differentiation $[13,14]$. Hematopoiesis in the lymph gland is regulated by a group of cells at the posterior end of the primary lobes, the posterior signaling center (PSC) $[13,15]$. The transcription factor Collier controls this homeostasis by coordinating the regulation of the cell number in the PSC. Early in embryonic development, Collier is expressed in all lymph gland cells, whereas in larvae its expression is restricted to the PSC [13].

The hematopoietic function of the sessile hematopoietic tissue was recently discovered $[3,10]$. It is a subepithelial compartment of hemocytes, which respond to immune induction by the oviposition of parasitic wasps, detaching and differentiating into lamellocytes $[3,10,16$, 17].

Foreign objects that are too large to be taken up by phagocytosis are isolated through the action of lamellocytes. Although much is already understood concerning the elimination of such particles, several aspects remain unexplained. To gain further insight into the underlying mechanisms of how multicellular organisms isolate foreign bodies, including the eggs of parasites, we analyzed the encapsulation reactions of different Drosophila species. In several species of the ananassae subgroup of Drosophilidae, we identified a previously undescribed cell type, the multinucleated giant hemocyte (MGH), and we therefore set out to perform a detailed analysis of this cell type in a representative species, Drosophila ananassae. We generated immunological and transgenic markers and used them to investigate the function of MGHs in naive and immune-challenged larvae. We next determined the tissue origin of giant blood cells in the main hematopoietic compartments, with special emphasis on the lymph gland, one of the hematopoietic tissues in $D$. melanogaster, the source of encapsulating lamellocytes.
The mechanism of the giant cell formation was analyzed by using nuclear labeling of hemocytes. Different species of parasitic wasps were used for infection, and the morphological features and immunological phenotypes of the blood cells were determined.

\section{Experimental Procedures}

\section{Drosophila Stocks}

The Drosophila species D. melanogaster, D. sechellia, D. erecta, D. persimilis, D. mojavensis, D. ficusphila, D. elegans, D. fuyamai, D. pallidosa, D. atripex, D. malerkotliana, D. parabipectinata, D. pseudoananassae, D. nikananu, D. vulcana, D. serrata, D. auraria, D. rhopaloa, D. mayri, D. bicornuta, D. biarmipes, D. lucipennis, D. mimetica, D. pseudotakahashii, D. lutescens, D. prostipennis, D. mauritiana, D. teissieri, D. pallidosa, D. simulans, D. willistoni, D. yakuba, D. ananassae, D. eugracilis, D. bipectinata, D. virilis and D. pseudoobscura were investigated (the stock identifiers are listed in online suppl. table S1; for all online suppl. material, see www. karger.com/doi/10.1159/000369618). The flies were kept on a standard cornmeal-yeast diet at $25^{\circ} \mathrm{C}$.

\section{Parasitic Wasps}

Leptopilina boulardi strain G486 [18], L. heterotoma strain Lh14 and L. victoriae strain L.v.UNK (kindly provided by Prof. Todd Schlenke) were used.

\section{Antibodies}

Primary Antibodies. 4H1 (mouse monoclonal antibody, tissue culture supernatant, against plasmatocytes of D. ananassae, used neat) and 7C5 (mouse monoclonal antibody, tissue culture supernatant, against MGHs of Drosophila ananassae, used neat) and anti-Atilla (L1 [19]) were produced in our laboratory. (These antibodies are freely available upon request from I.A.) Anti-Collier (mouse monoclonal antibody, 1:100 dilution) was kindly provided by Dr. Michèle Crozatier). Phalloidin-rhodamine, anti-tubulin (mouse monoclonal antibody, 1:30 dilution), anti-bromodeoxyuridine (BrdU; Alexa Fluor 488-conjugated mouse monoclonal antibody, 1:10 dilution) and anti-GFP (rabbit polyclonal antibody, 1:2,000 dilution) were from Invitrogen; anti-phospho-histone H3 (rabbit polyclonal antibody, 1:10,000 dilution) was from Sigma.

Secondary Antibodies. Anti-mouse Alexa Fluor 488 (goat antibody, 1:1,000 dilution), anti-mouse Alexa Fluor 568 (goat antibody, 1:1,000 dilution) and anti-rabbit Alexa Fluor 488 (goat antibody, 1:1,000 dilution) were from Invitrogen.

\section{Production of Monoclonal Antibodies}

The immunization with hemocytes and the hybridoma production were carried out as described [20]. Briefly, female BALB/c mice were immunized three times at 3 -week intervals with $10^{6}$ hemocytes from $L$. bolulardi-infected $D$. ananassae larvae. The hemocytes used for immunization were isolated $72 \mathrm{~h}$ after infection. Three days after the third immunization, mouse spleen cells were fused with $\mathrm{Sp} 2 / 0$ cells in the presence of polyethylene glycol (PEG-1540). Hybridomas were selected in HAT medium as described by Köhler and Milstein [21] and screened for antibody production on acetone-fixed hemocyte smears from wasp-infected D. ananassae larvae. 


\section{Generation of Transgenic Lines in D. ananassae}

We used two PiggyBac-based transformation vectors to test the expression pattern of the cloned promoter fragments. PB-iehrmCherry-EGFP driver plasmid was engineered so as to have an $m$ Cherry fluorescent reporter gene driven by a strong baculovirus promoter, the IE- 1 and the hr 5 enhancer. This vector also had an attB donor sequence so that it could be integrated into the host genome by both PiggyBac transposase and phiC 31 integrase-directed transformation processes. The map and full sequence of the vector is presented as online supplementary material. The DNA fragment used as $\mathrm{Hml}$ promoter includes 2,952 bp from the D. melanogaster genome (3L:13836355-3L:13839307) located upstream of the start codon of the $\mathrm{Hml}$ gene. This fragment was cloned upstream of the EGFP into the AscI-PmeI (blunt) site of the PB-iehr-mCherry-EGFP driver plasmid, and the construct was used for the generation of $D$. ananassae transgenic stocks. The Pxn promoter used was a 1,621 bp fragment (2R:9450688-2R:9452309) of the D. ananassae genome, located upstream of the Pxn gene (GF10247) and cloned into the AscI-PmeI (blunt) site of the PB-iehr-mCherry-EGFP driver plasmid (online suppl. fig. S7, the sequences are presented in the online supplementary data). As we encountered some problems during the detection of the double ( $m$ Cherry and EGFP) fluorescence, we transferred the Pxn-promoter-EGFP-K10-polyA AscI-FseI fragment into the unique NheI restriction site of the PiggyBac_PB (http://www. ncbi.nlm.nih.gov/nuccore/AY515146) vector after its NheI site was converted to AscI-FseI through a synthetic linker. This vector had a mini-white marker gene instead of $m$ Cherry. The construct obtained was used for the generation of $D$. ananassae transgenics.

The PiggyBac plasmids together with the helper plasmid were injected into $D$. ananassae embryos. Male flies arising from the embryos injected with the Hml-GFP $m$ Cherry and the Pxn-GFP mini-white plasmids were crossed to wild-type $D$. ananassae and white mutant $D$. ananassae virgin females, respectively. Transformants were selected according to the expression of the mCherry and the mini-white reporter gene. Homozygous transgenic lines were generated by crossing males and virgin females that showed a high level of expression of the marker gene.

\section{Indirect Immunofluorescence}

Hemocytes and cuticle preparations were fixed with acetone for $6 \mathrm{~min}$, or with $2 \%$ paraformaldehyde for $12 \mathrm{~min}$, and blocked with $0.1 \%$ BSA in PBS for $20 \mathrm{~min}$. In the case of the cuticle preparations, we used $0.1 \%$ BSA in PBS supplemented with $0.01 \%$ Triton $\mathrm{X}-100$. The samples were incubated with the primary antibodies for $1 \mathrm{~h}$, washed 3 times for $5 \mathrm{~min}$ in PBS, incubated with secondary antibodies for $45 \mathrm{~min}$, washed 3 times for $5 \mathrm{~min}$ and covered with Fluoromount $\mathrm{G}$ medium and coverslip. Six parallel samples were prepared for each staining and over 500 cells were scored in each. The experiments were repeated three times. The samples were analyzed with a Zeiss Axioskope 2MOT epifluorescent or an Olympus FV1000 confocal microscope.

\section{Preparation of the Lymph Gland}

Larvae were opened along a longitudinal axis, and the digestive tract and the fat body were removed. The carcasses were then fixed with $2 \%$ paraformaldehyde for $12 \mathrm{~min}$, washed 3 times for $5 \mathrm{~min}$ in PBS and blocked with $0.1 \%$ BSA in PBS supplemented with $0.01 \%$ Triton X-100 for $20 \mathrm{~min}$. The samples were incubated with the primary and secondary antibodies as described above. Analyses were performed with an Olympus FV1000 confocal microscope.

\section{Colchicine Treatment of Hemocytes}

Ninety-six hours after immune induction, hemocytes were isolated from larvae on microscopic slides (Hendley-Essex) in $30 \mu \mathrm{l}$ of Drosophila-Schneider's medium supplemented with 5\% FCS and settled for $10 \mathrm{~min}$ at room temperature; then, the medium was replaced by Schneider's medium containing colchicine $(0.4 \mathrm{mg} /$ $\mathrm{ml})$. After a 45 -min incubation at room temperature, cells were fixed with $4 \%$ paraformaldehyde for $10 \mathrm{~min}$ and washed 3 times for $5 \mathrm{~min}$ in PBS, pH 7.2, followed by fixation in methanol for 20 min at $-20^{\circ} \mathrm{C}$ and blocking for $1 \mathrm{~h}$ in $0.1 \%$ BSA-PBS at room temperature. After a 1-hour incubation with anti-tubulin antibody, the samples were washed 3 times for $5 \mathrm{~min}$ each in PBS, next incubated with the secondary antibody and DAPI (Sigma, 1:400 dilution) for $45 \mathrm{~min}$, washed 3 times for $5 \mathrm{~min}$ in PBS and covered with a coverslip in Fluoromount $\mathrm{G}$ medium (SouthernBiotech).

\section{Videomicroscopy}

Hemocytes were collected $72 \mathrm{~h}$ after immune induction and filmed for $30 \mathrm{~min}$ at room temperature in $200 \mu \mathrm{l}$ of DrosophilaSchneider's medium, using a Sony DX high-definition camera.

\section{In vivo Confocal Microscopy}

Third instar larvae were paralyzed in a solution of Dichlorvos (Fluka) in Drosophila Ringer's solution (1:1,000) for 5 min at room temperature [22]. Larvae were then mounted on glass-bottomed dishes (Cell E\&G) coated with glue and covered with Voltalef 10S oil (VWR). The samples were analyzed with a Leica TCS SP5 II confocal microscope. The images were stacked and sequenced into video format by using ImageJ [22].

\section{Hemocyte Counting}

Second instar $D$. ananassae larvae were exposed to different female parasitic wasps (L. boulardi, L. heterotoma and L. victoriae) for $8 \mathrm{~h}$. Hemocytes were isolated from 10 infected larvae 24, 48 and $72 \mathrm{~h}$ after infection. Individual larvae were bled in $30 \mu \mathrm{l}$ of Schneider's medium supplemented with 5\% FCS on multispot microscope slides (Hendley-Essex). Cells were allowed to settle at room temperature for $45 \mathrm{~min}$, fixed with acetone for $6 \mathrm{~min}$ and immunostained with the monoclonal antibodies, as described above. Uninduced controls were treated in parallel. Nuclei were visualized with DAPI.

\section{Parasitization Assay}

Forty-second instar larvae were transferred into a Petri dish containing standard fly food and were exposed to 8 female wasps for $4 \mathrm{~h}$. Infected larvae were selected on the basis of the hemolymph coagulate at the site of the injury caused by the ovipositor of the wasp and used for further experiments. For survival assays, 30 infected larvae were selected and then transferred into vials and allowed to eclose (as described by Mortimer et al. [5]). The numbers of eclosed flies and wasps were determined. The experiment was repeated four times.

\section{BrdU Labeling of the Larvae and Detection of Cell Fusion ex} vivo

A $93 \mathrm{~mm}$ BrdU stock solution was prepared in $50 \%$ ethanol and stored at $-20^{\circ} \mathrm{C}$. D. ananassae flies were allowed to lay eggs for $24 \mathrm{~h}$ at $25^{\circ} \mathrm{C}$ in the dark on vials containing standard fly food supplemented with $1 \mathrm{~mm}$ BrdU, and larvae were then kept on the vials. Second instar larvae were infected with female parasitoid wasps 
(L. boulardi) overnight in the dark. Hemocytes were isolated from the larvae $48 \mathrm{~h}$ after parasitization. In the case of the 4-hour labeling, infected larvae were transferred to vials containing the standard fly food supplemented with $1 \mathrm{~mm}$ BrdU. Hemocytes of BrdU-treated and untreated larvae were mixed in $30 \mu \mathrm{l}$ of Schneider's medium supplemented with 5\% FCS on multispot microscope slides (Hendley-Essex). Cells were allowed to settle at room temperature for 45 $\mathrm{min}$, fixed with acetone for $6 \mathrm{~min}$, treated with $2 \mathrm{M} \mathrm{HCl}$ for $15 \mathrm{~min}$, washed 4 times in PBS and blocked in PBS containing $0.1 \%$ BSA for $20 \mathrm{~min}$. The samples were incubated with mouse anti-BrdU Alexa Fluor 488-conjugated antibody for $1 \mathrm{~h}$ and next washed 3 times in PBS; the reaction was amplified with a goat anti-mouse Alexa $488 \mathrm{Ig}$ incubation for $45 \mathrm{~min}$. Nuclei were visualized with DAPI (Sigma, 1: 400). The slides were washed 3 times in PBS, covered with Fluoromount $\mathrm{G}$ medium (SouthernBiotech) and analyzed with an epifluorescence microscope (Zeiss Axioskope 2 MOT).

In vivo Phagocytosis Assay

D. ananassae larvae were injected with FITC-labeled Escherichia coli bacteria ( $5 \mu \mathrm{l}$ pelleted, FITC-labeled bacteria in $1 \mathrm{ml}$ PBS) 24,48 and $72 \mathrm{~h}$ after infection. Hemocytes were isolated $40 \mathrm{~min}$ after injection, fixed and reacted with antibodies as described above. The phagocytic capacity of hemocytes was correlated with their immunological phenotype. Analysis was performed with an Olympus FV1000 confocal microscope.

\section{Electron Microscopy}

For electron microscopy, the hemolymph of parasitized larvae was collected and fixed in a solution of $1 \%$ glutaraldehyde and $1 \%$ paraformaldehyde in $0.1 \mathrm{M}$ phosphate buffer $(\mathrm{pH} 7.4)$ for $2 \mathrm{~h}$. Following centrifugation and washing in phosphate buffer, cells were postfixed in $1 \%$ osmium tetroxide. The samples were dehydrated in graded series of ethanol and flat-embedded in Spurr. Ultrathin sections were mounted on Formwar-coated one-hole grids, stained with lead citrate and studied in a Zeiss CEM 902 electron-filtering electron microscope.

Wounding of Larvae and Melanization Assay

For cuticle wounding, third instar larvae were collected, washed in Drosophila Ringer solution and air-dried on microscopic slides. They were punctured at the posterior end with minutien needles (0.15 diameter; Austerlitz Insect Pins) and removed on filter paper into wet chambers for $90 \mathrm{~min}$. The melanized punctures on the larvae were examined with a Leica MZ FL III UV stereo microscope.

\section{Results}

\section{Identification of $\mathrm{MGHs}$}

We studied circulating hemocytes of several Drosophila species (see Experimental Procedures and online suppl. table S1) and found that infection with the parasitoid wasp $L$. boulardi is followed by the appearance of a previously unidentified giant cell in the hemolymph of several species of the ananassae subgroup of Drosophilidae, including $D$. ananassae, D. pallidosa, D. atripex, D. pseudoanassae, D. bipectinata, D. malerkotliana and D. parabipecinata (online suppl. fig. S1). These giant cells, which vary in size from 40 to several hundred micrometers, can attach to glass. They have several nuclei in their cytoplasm (fig. 1). Ex vivo video analysis revealed that they move actively, spread vigorously on a glass surface and are capable of recognizing parasitic eggs and of wrapping around them, showing that they play a role in capsule formation (online suppl. video S1). These cells are not present in the embryo and in the adult (not shown) but fully develop in immune-induced larvae 48-96 h after the oviposition of parasitic wasps. To characterize this cell type further, we chose $D$. ananassae as a representative species in view of its sequenced and annotated genome and its genetic background.

\section{Morphological, Immunological and Functional \\ Characteristics of $\mathrm{MGH}$}

Transmission electron microscopic analysis of fully developed (96-hour) MGHs revealed that these cells are syncytia with a complex network of membrane extensions that interconnect cytoplasmic bundles, without separating cell membranes (fig. 1b). Cytoskeletal staining of MGHs with TRITC-phalloidin and anti-tubulin antibodies revealed dense, continuous actin fibers and interconnecting microtubules among several, randomly located nuclei in the cytoplasm (fig. 1c), implying that nuclei are not separated from each other. Isolated MGHs unfold vigorously on a glass slide. Their unfolding is blocked by colchicine treatment, demonstrating the necessity of microtubule polymerization for this event (fig. 1d).

In order to identify and track MGHs, we developed immunological reagents for hemocytes of $D$. ananassae. From a panel of monoclonal antibodies, two discriminative antibodies, $4 \mathrm{H} 1$ and 7C5, were selected. The $4 \mathrm{H} 1$ antibody reacts with small spherical phagocytic cells (fig. 2a) with a diameter of 5-10 $\mu \mathrm{m}$, which comprise the bulk of cells in the circulation in naive larvae. With these criteria, we defined $4 \mathrm{H} 1$ as an antibody to plasmatocytes. The 7C5 antibody reacts specifically with nonphagocytic MGHs (fig. 2b) and with small filariform cells in the hemolymph of immune-induced larvae.

MGHs are not present in naive larvae, but appear after oviposition by the parasitoid wasp L. boulardi. Immunostaining of parasite eggs for the discriminative immunological markers revealed that 24 and $48 \mathrm{~h}$ after immune induction, plasmatocytes adhere to the egg (not shown), and by $72 \mathrm{~h}$, parasites are encapsulated by both plasmatocytes and MGHs (fig. 2b). The capsule is not melanized. Mechanical wounding of larvae with an insect pin induces melanization similar to that observed in D. melanogas- 
ter, showing that the melanization cascade in this species is not impaired (online suppl. fig. S2).

Previous studies on $D$. melanogaster $[10,23]$ revealed that phagocytic plasmatocytes convert to nonphagocytic lamellocytes, and cells in an intermediate stage of differentiation display the characteristics of both cell types. These
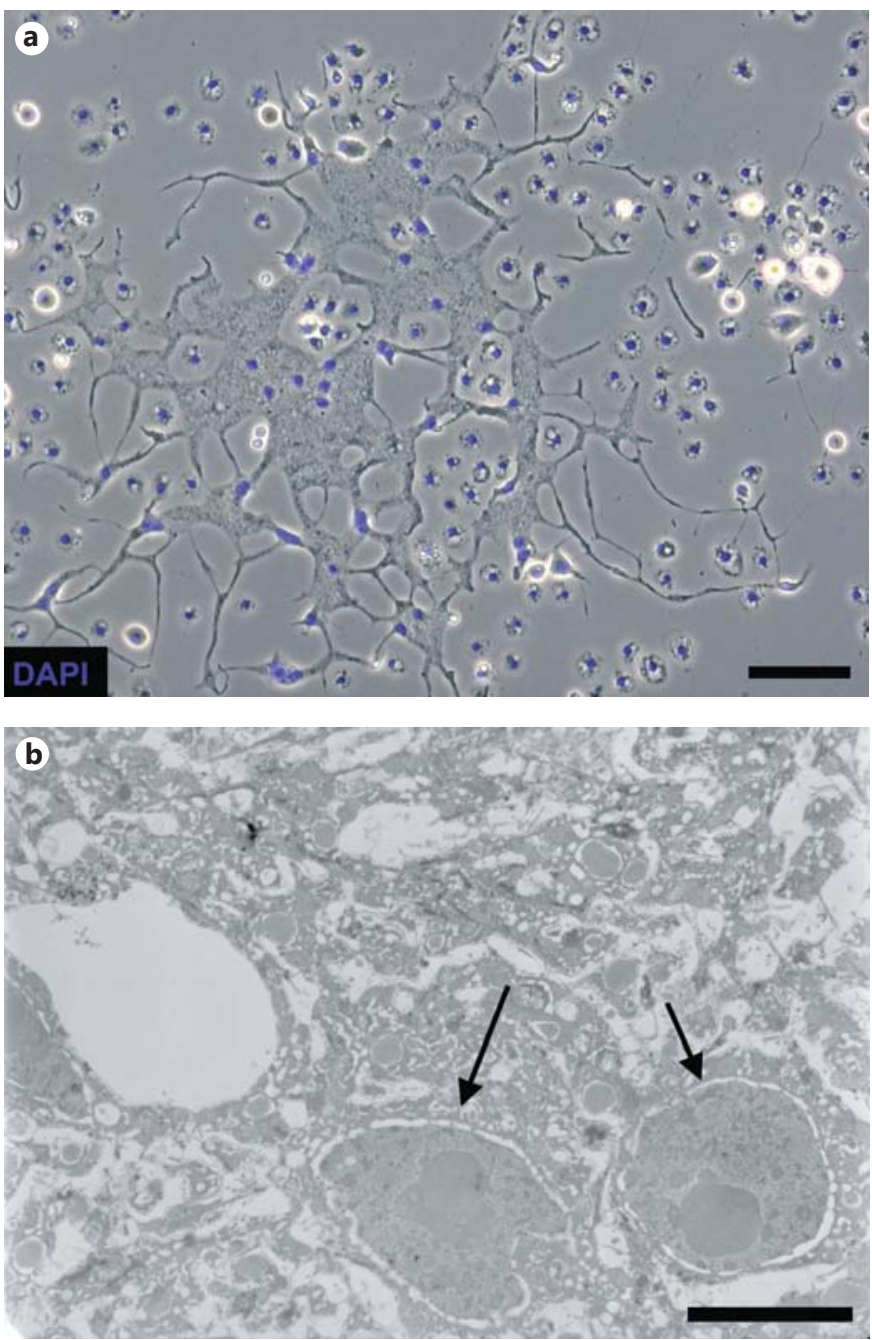

Fig. 1. Morphological analysis of MGHs. a Phase-contrast microscopic image of an MGH. b Transmission electron microscopic cross-section of an MGH. The arrows indicate the nuclei. c TRITCphalloidin staining (red) of hemocytes from immune-induced

Fig. 2. MGHs are not phagocytic but are required for the encapsulation reaction. Cell-type-specific staining (red) of MGHs (7C5) and plasmatocytes (4H1). a Phagocytosis of FITC-labeled E. coli (green) by $D$. ananassae plasmatocytes. b Encapsulation of the parasite larvae (6 larvae were immunostained and analyzed for each antibody, the experiment was repeated 3 times). c A filariform data prompted us to investigate the early stages of $\mathrm{MGH}$ differentiation in D. ananassae. Twenty-four hours after parasitoid immune induction, we observed bipolar mononuclear phagocytic cells expressing the 7C5 marker (fig. 2c). This indicates that these cells are in an intermediate stage of transition from phagocytic cells to encapsulating MGHs.
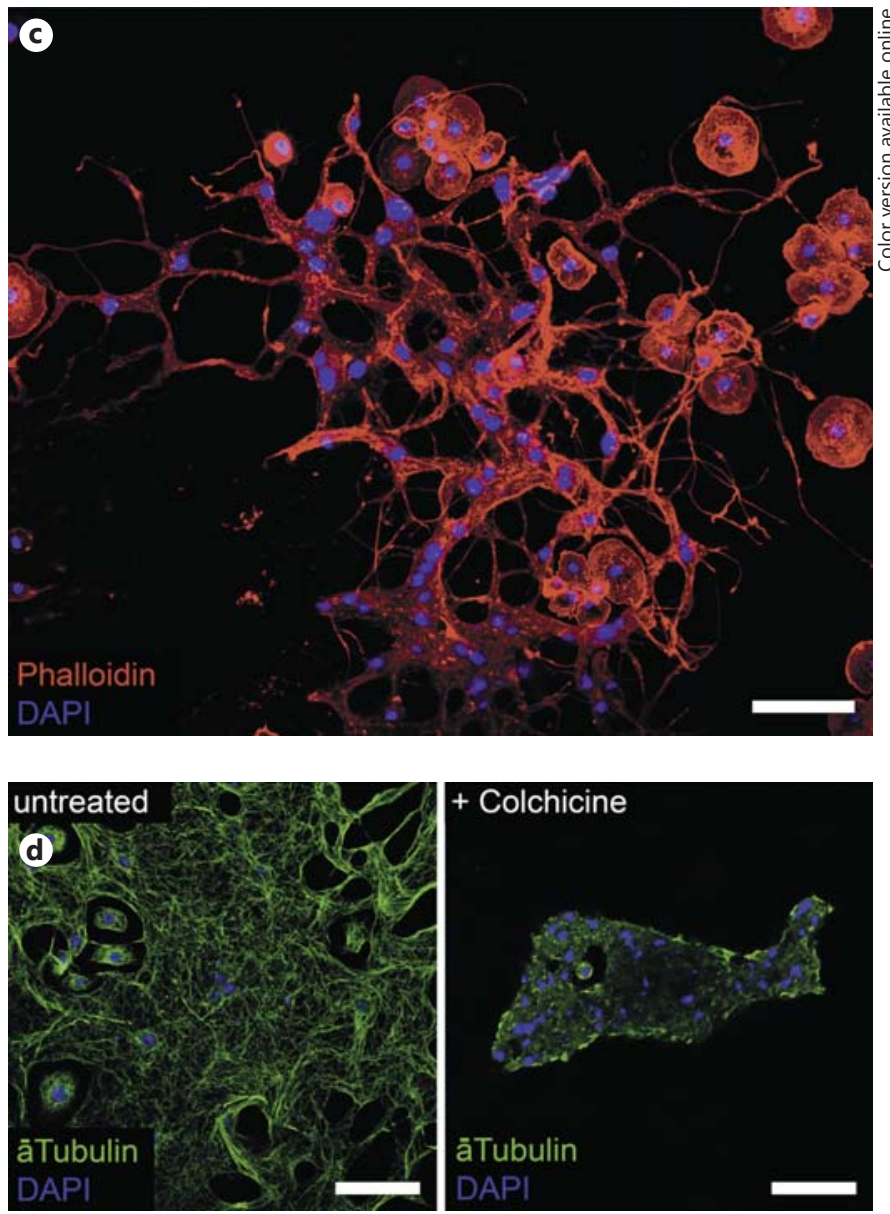

D. ananassae larvae. d Anti-tubulin (green) and nuclear DAPI staining (blue) of the untreated and colchicine-treated MGHs of immune-induced larvae. Scale bars = 5 (b), 25 (d) and $50 \mu \mathrm{m} \mathrm{(a,} \mathrm{c;}$ see online version for colors).

hemocyte from an immune-challenged larva, stained by MGHspecific antibody (7C5, red). The phagocytic capacity is indicated by the presence of FITC-labeled E. coli (green) within the cell. Representative pictures were selected from 3 independent experiments, with 5 animals in each group. Scale bars $=50 \mu \mathrm{m}$ (see online version for colors).

(For figure see next page.) 

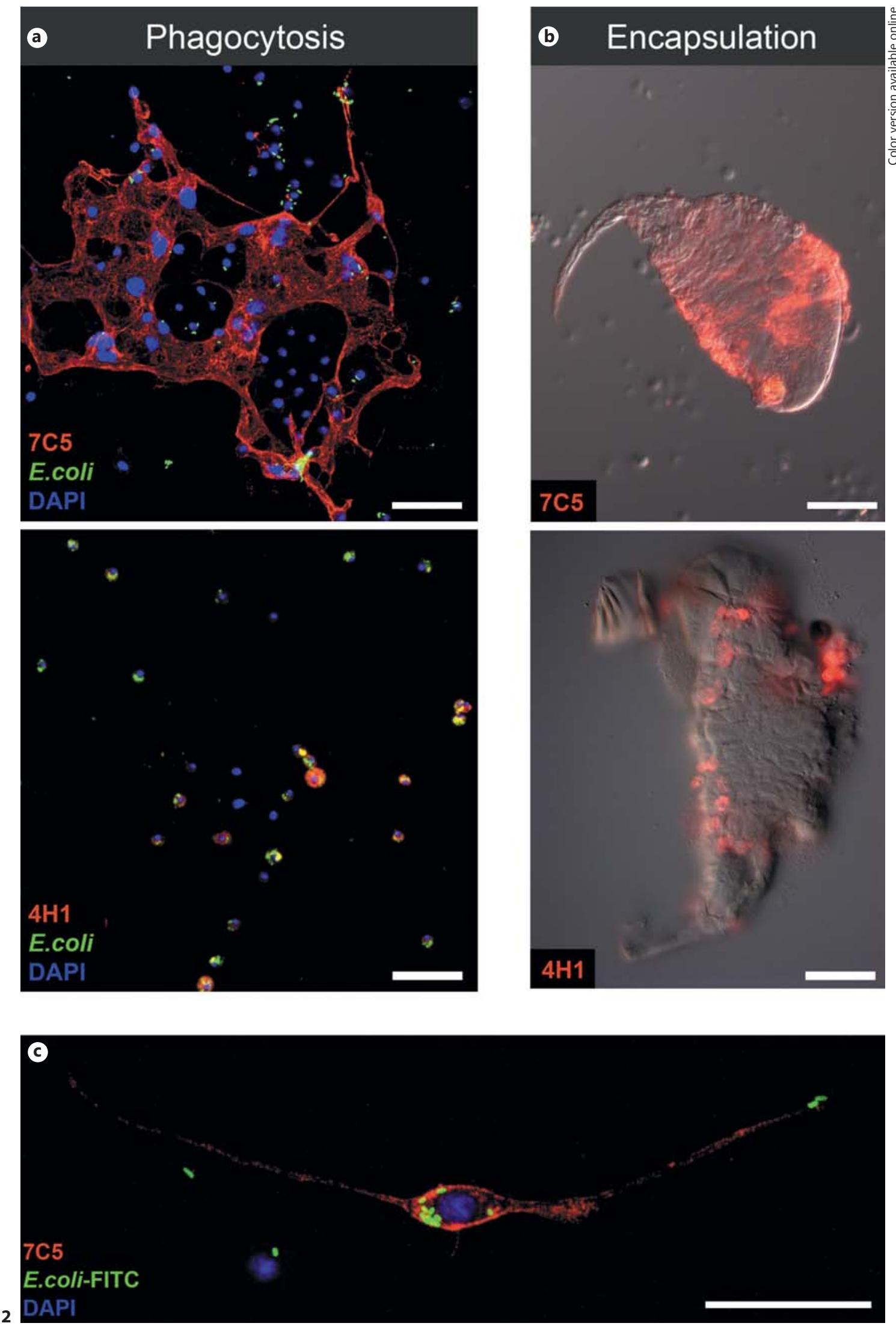

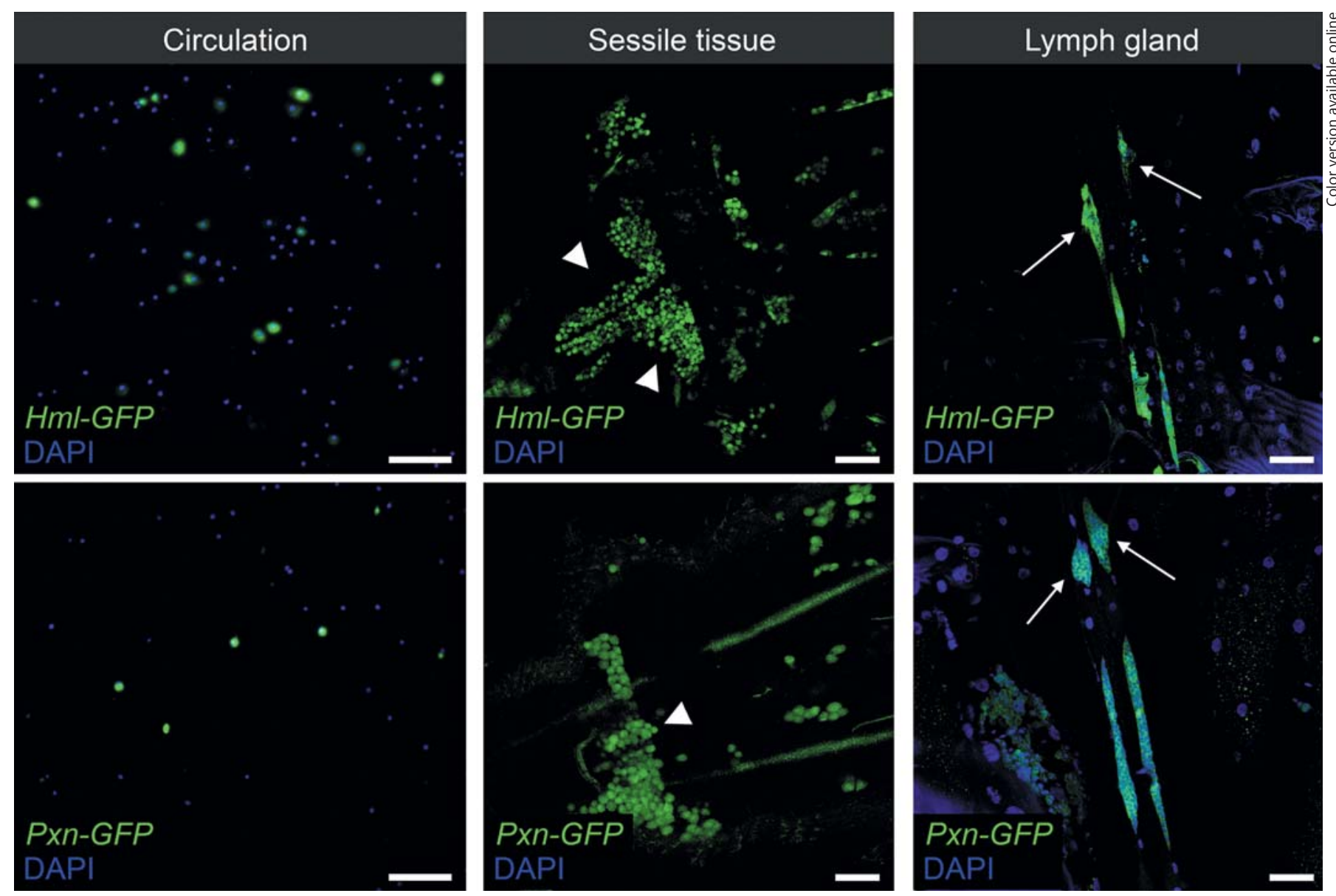

Fig. 3. Analysis of the hemocyte compartments of the D. ananassae larva with in vivo reporters. The hemocytes expressing the $\mathrm{Hml}$ GFP and Pxn-GFP transgenes can be seen in green (see online version for colors). The arrows indicate the primary lymph gland

\section{Hematopoietic Compartments of D. ananassae}

For a detailed characterization of the hemocyte types and hematopoietic compartments, we created hemocytespecific transgenic reporter constructs. The upstream regulatory sequences of the Hemolectin and Peroxidasin genes were fused with the EGFP reporter gene. The $\mathrm{Hml}$ regulatory region [24] was obtained from $D$. melanogaster, while the regulatory region of the Peroxidasin gene [25] was identified by sequence homology between $D$. melanogaster and D. ananassae. Homozygous lines were established from both transgene insertions (referred to below as Hml-GFP and Pxn-GFP).

Analysis of the hematopoietic compartments in the transgenic stocks revealed that the transgenes were expressed in the hemocytes of the third instar larvae. Expression was detected in $10-20 \%$ of the circulating hemocytes (fig. 3). The sessile hematopoietic compartment was detected as aggrega- lobes, and the arrowheads show the sessile islets. The pictures were selected from 3 independent experiments, 4 larvae were used in each group. Scale bars $=50 \mu \mathrm{m}$.

tions of hemocytes in a striped pattern in the subepithelial tissues (fig. 3), similarly to D. melanogaster [16]. In the lymph gland, every cell in the primary and secondary lobes expressed the transgenes. This expression pattern is strikingly different from that of $D$. melanogaster, where the activity of these plasmatocyte-specific elements is restricted to the secondary lobes and to the cortical zone of the primary lobes. Moreover, the primary lymph gland lobes of the naive third instar $D$. ananassae larvae were much smaller than their D. melanogaster counterparts and the structure of the lymph gland appeared to be homogeneous, without obvious separation of cortical and medullary zones (fig. 3).

\section{Compartmental Origin of MGHs}

It has been established that all the hematopoietic compartments in D. melanogaster contribute to the antiparasite immune response $[3,10]$. To determine the roles of 
the hematopoietic compartments in the differentiation of MGHs, we monitored the phenotypic changes in hemocytes in the circulation, the sessile hematopoietic tissue and the lymph gland in parasitized $D$. ananassae larvae. Time lapse analysis demonstrated that blood cells carrying the 7C5 marker first appear in the circulation $24 \mathrm{~h}$ after the immune induction (fig. 4a). These cells are mononuclear hemocytes with bipolar morphology. Forty-eight hours after immune induction, they appear in the sessile hematopoietic tissue, as revealed by both immunostaining with 7C5 antibody (fig. 4a) and by in vivo time lapse confocal microscopic analysis of the sessile tissue in immobilized [22] Hml-GFP larvae (online suppl. video S2). The number and size of MGHs in the circulation peaked 72-96 h after induction (online suppl. fig. S3).

Following the immune induction, the lymph gland was slightly swollen, but 7C5-expressing hemocytes were not found in the lobes, and the organ did not disintegrate (fig. 4a). The fractions of dividing cells revealed in the lymph glands in uninduced and immune-induced larvae by anti-phospho-histone $\mathrm{H} 3$ staining were the same (online suppl. fig. S4), indicating that the lymph gland of $D$. ananassae does not serve as a source of MGHs in the antiparasite immune response, which is in sharp contrast with the immune response and hemocyte differentiation in $D$. melanogaster $[10,12,13]$. To gain insight into the structure of the lymph gland and why this organ does not contribute to the MGH pool, we checked the expression of transcription factor Collier, recognized as one of the key regulatory factors in hemocyte development in the lymph gland [13]. In D. melanogaster, a small number of cells at the posterior tip of the primary lobes, the PSC, exhibited Collier staining. In contrast, in D. ananassae, the majority of the lymph gland cells express the Collier transcription factor in both naive and immune-induced larvae (fig. $4 \mathrm{~b}$ ). In D. ananassae, we could not confirm heterogeneity with respect to Collier expression or to the expression of the plasmatocyte markers Hml-GFP and $P x n-G F P$, and we could not identify structural and functional regions/zones within the primary lobes.

\section{Mechanism of MGH Formation}

Since the transmission electron microscopic analysis and studies on the cytoskeletal structure indicated that MGHs contain several nuclei without separating cell membranes, we considered whether these syncytia are formed by the division of nuclei (endomitosis) or by cell fusion. MGHs of Hml-GFP larvae displayed a patchy GFP pattern, indicating that these cells were formed by the fusion of hemocytes expressing the Hml-GFP trans- gene with other hemocytes that lack GFP expression (fig. 5a).

In order to investigate whether nuclear division plays a role in MGH formation, the expression of a mitosis marker, phospho-histone $\mathrm{H} 3$, was first assessed by antiphospho-histone $\mathrm{H} 3$ staining of hemocytes isolated $48 \mathrm{~h}$ after an immune challenge. Dividing cells were found among plasmatocytes (online suppl. fig. S4) in each hemocyte compartment, and no staining of MGHs was detected. As anti-phospho-histone $\mathrm{H} 3$ staining marks only recent mitotic events, we also used an approach involving the labeling of DNA with the nucleotide analogue BrdU in cells passing through the S-phase, followed by immunostaining for the incorporated BrdU. The results showed that in the course of a 4-hour pulse period, $70 \%$ of plasmatocytes were labeled, while no labeling was seen in MGHs (online suppl. fig. S5), confirming that MGH nuclei did not divide. Although these two approaches are based on different principles, both indicate that MGHs are not formed by internal nuclear division but rather by cell fusion. To verify that MGHs are formed by cell fusion, hemocytes of immune-induced larvae were labeled in vivo with BrdU, which resulted in $>95 \%$ nuclear labeling, and BrdU-labeled hemocytes were mixed with unlabeled hemocytes ex vivo. Microscopic analysis revealed that MGHs contained both labeled and unlabeled nuclei in the same cytoplasm (fig. 5b), confirming that they were formed by cell fusion. The fusion of MGHs was also investigated by time lapse video analysis of hemocytes of immune-induced larvae (online suppl. video S3). It emerged that MGHs underwent complete fusion within $45 \mathrm{~min}$.

In order to investigate if plasmatocytes contribute to the formation of MGHs or the fusion occurs among activated blood cells, hemocytes, isolated from BrdU-labeled uninduced and unlabeled immune-induced larvae were mixed ex vivo. Immunostaining of nuclei for the incorporated BrdU revealed that nuclei of MGHs did not contain the nucleotide analogue (online suppl. fig. S6), showing that MGHs were formed by the fusion of activated blood cells, and nonactivated plasmatocytes do not contribute to this process.

\section{Suppression of MGH Formation by Parasitic Wasps}

Studies of the $D$. melanogaster encapsulation response revealed that several species of parasites can escape the cellular immune response by producing factors that interfere with the differentiation of lamellocytes $[8,26]$. To determine whether parasites can similarly inhibit the differentiation of MGHs, we infected second instar $D$. ananassae 

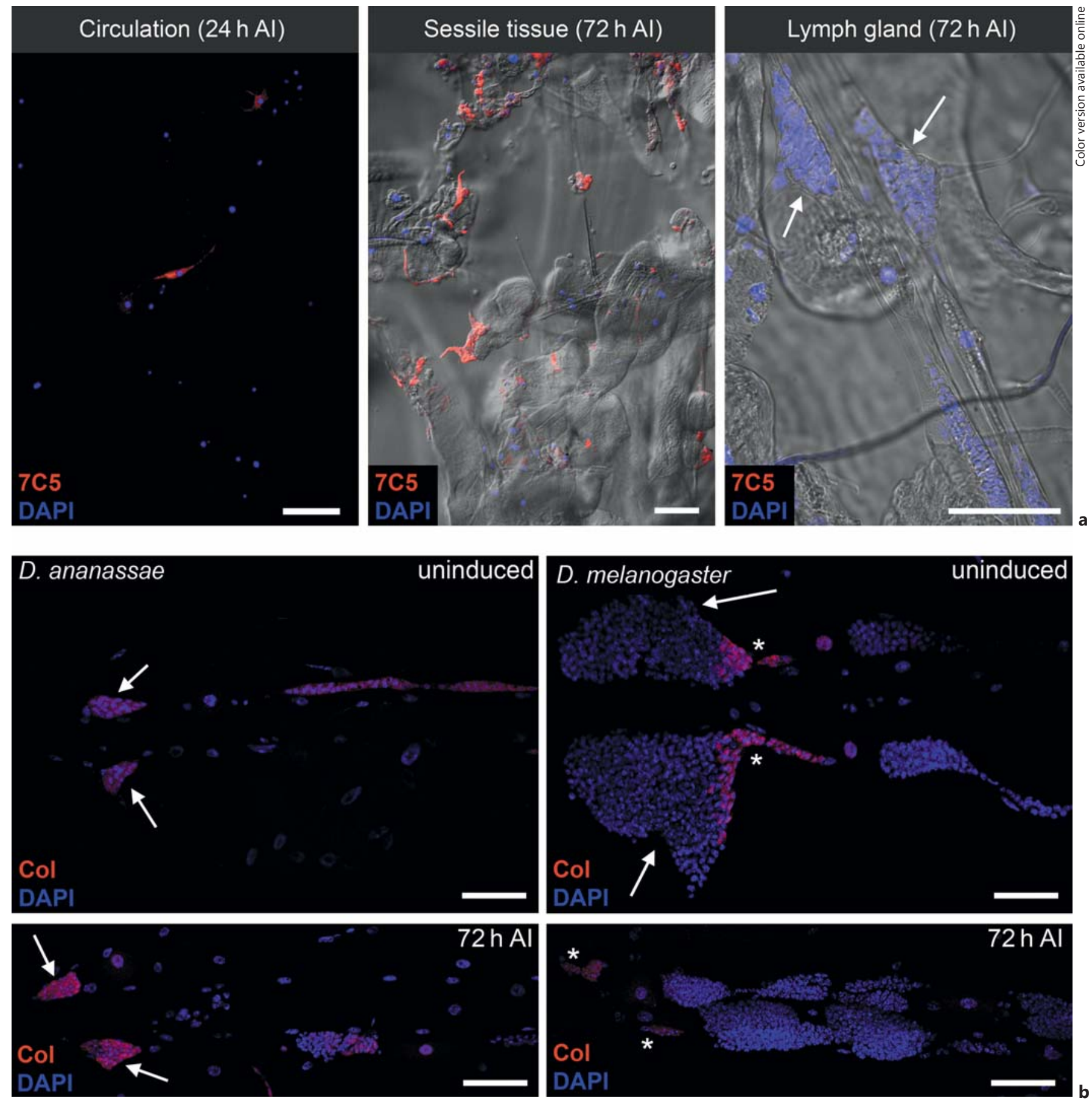

Fig. 4. The compartmental origin of MGHs. a The presence of MGHs in the larval hematopoietic compartments after infestation (AI) is revealed by cell type-specific staining (red). The arrows indicate the primary lobes of the lymph gland. b Collier staining (Col; red) of D. ananassae and D. melanogaster lymph glands. The

arrows indicate the primary lobes, while the asterisks show the PSC. The pictures were selected from 3 independent experiments, with 4 larvae used in each group. Scale bars $=50 \mu \mathrm{m}$ (see online version for colors). 

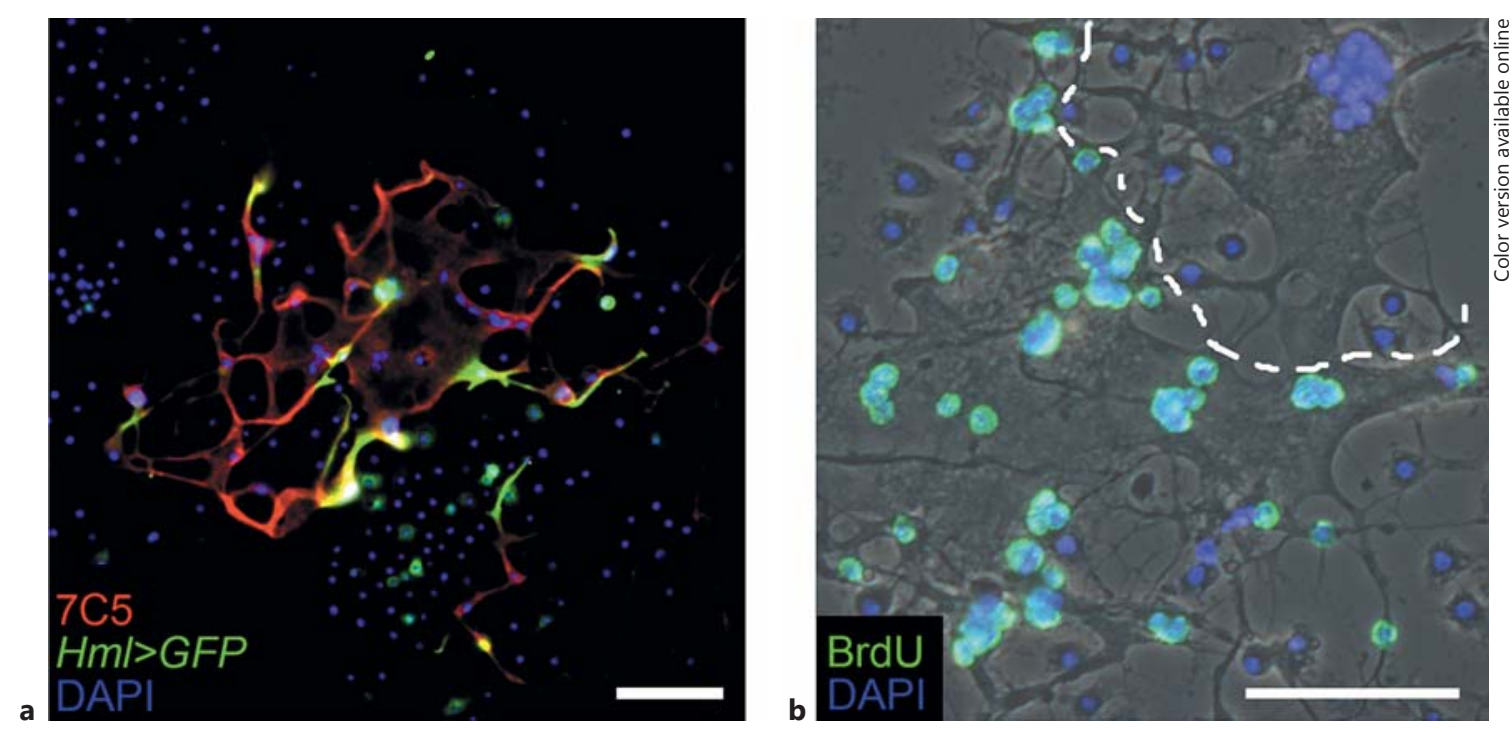

Fig. 5. The formation of MGHs. a Inhomogeneous GFP signal (green) within the MGHs (red). b Fusion of BrdU-labeled and unlabeled MGHs. The incorporation of BrdU is indicated by anti-Br-
$\mathrm{dU}$ antibody staining (green). The dashed curve shows the presumed position of the fusion site. Scale bars $=50 \mu \mathrm{m}$ (see online version for colors).
Fig. 6. a The immune response of D. ananassae larvae to different parasitoid wasps. The total number of hemocytes (y-axis) at different time points after immune induction (x-axis) are represented by the colored lines. The error bars indicate the standard error of the mean.

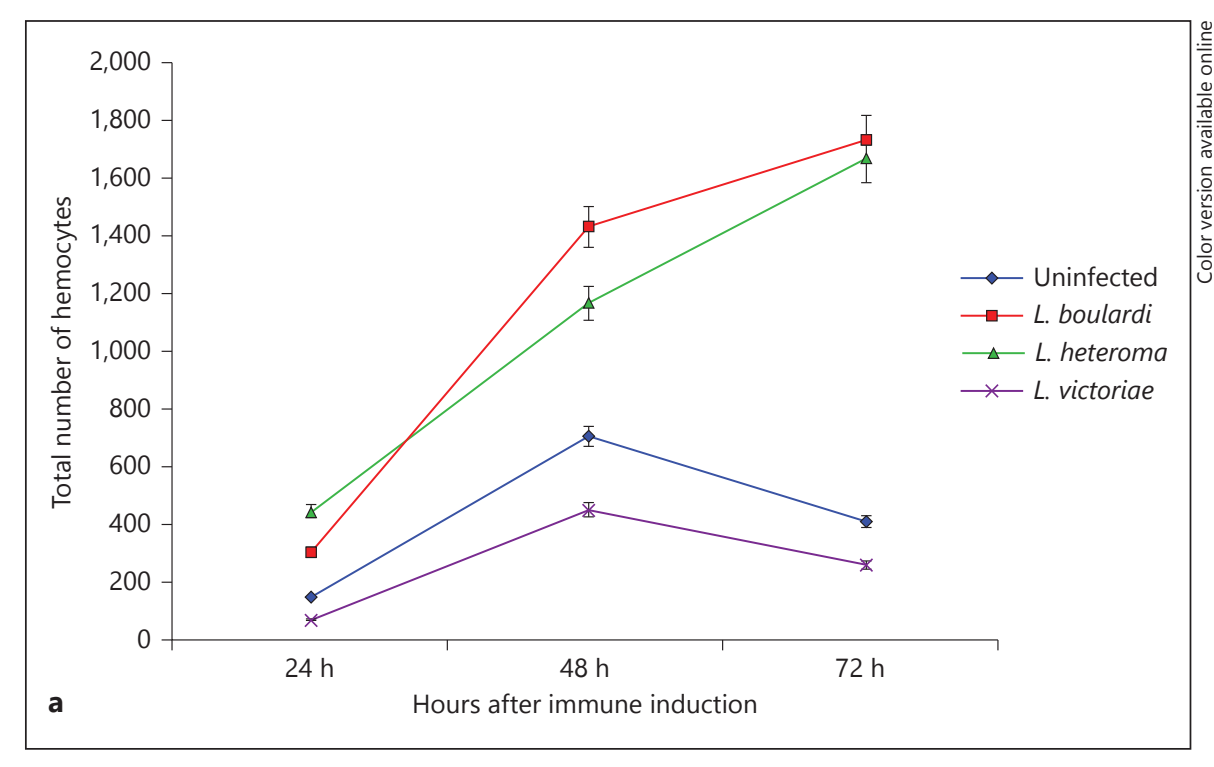

larvae with different species of parasitoid wasps. Infection by L. boulardi (a specialist for the melanogaster subgroup [27]) resulted in high hemocyte numbers and terminally differentiated MGHs. After infection with the generalist wasp L. heterotoma [28], proliferation of plasmatocytes was observed, and the differentiation of MGHs was impaired compared with that seen after infestation by L. boulardi. Interestingly, infection with another generalist wasp, L. victoriae [29], resulted in a lower total hemocyte number compared with that for either L. boulardi-infected or uninduced control larvae (fig. 6a). Moreover, the appearance of MGHs in the circulation was suppressed (fig. 6b). These results imply that the natural parasites of $D$. ananassae ( $L$. heterotoma and $L$. victoriae) actively suppress the immune reaction of the host by inhibiting hemocyte proliferation and the differentiation of MGHs. This immunosuppression also resulted in the increased success of parasitosis: while no $L$. boulardi wasp ever hatched from parasitized $D$. 

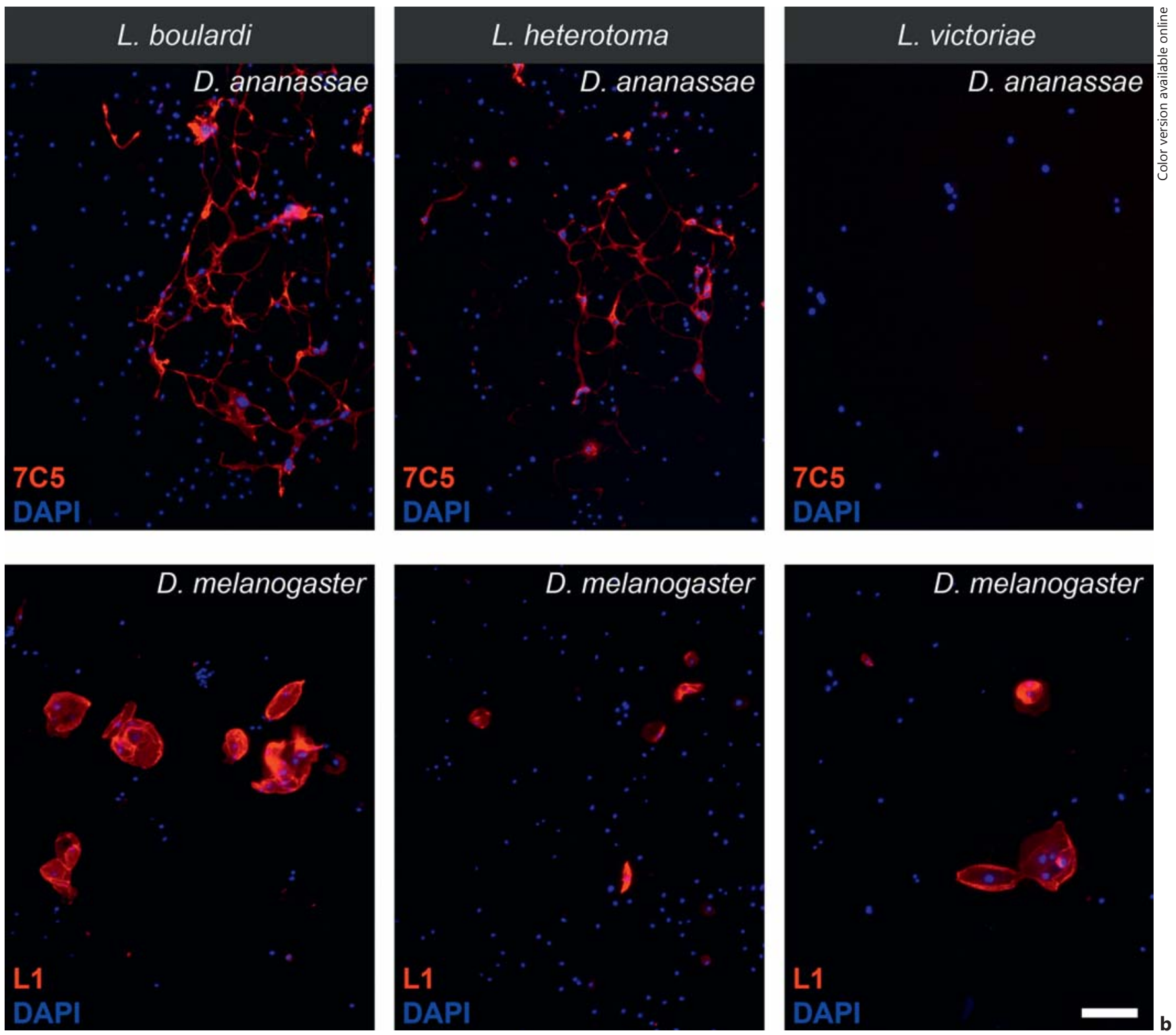

Fig. 6. b The immune response of $D$. ananassae larvae to different parasitoid wasps. The capsule-forming cells of $D$. ananassae and D. melanogaster $72 \mathrm{~h}$ after immune induction with different parasitic wasps. MGHs of D. ananassae and the lamellocytes of $D$. me-

ananassae pupae, L. heterotoma adults developed in $6 \%$ of the cases; L. victoriae parasitosis was even more successful: $43 \%$ of parasitized $D$. ananassae failed to eliminate the wasp larva. The infection of D. melanogaster with L. boulardi or L. victoriae wasps resulted in normal lamellocyte formation (fig. 6b), whereas we observed bipolar lamellocytes after the infection with L. heterotoma (fig. 6b) similarly to those described by Rizki et al. [8]. lanogaster are indicated by the red staining of cell type-specific monoclonal antibodies (7C5 and L1, respectively). Scale bar = $50 \mu \mathrm{m}$ (see online version for colors).

\section{Discussion}

The MGH, a unique effector cell type in innate immunity, was found in the ananassae subgroup of the Drosophila genus. These cells are not present in the embryo or in the adult, but appear in the hemolymph after parasitization with parasitic wasps. They can be recognized on the basis of their distinctive morphological features, the 


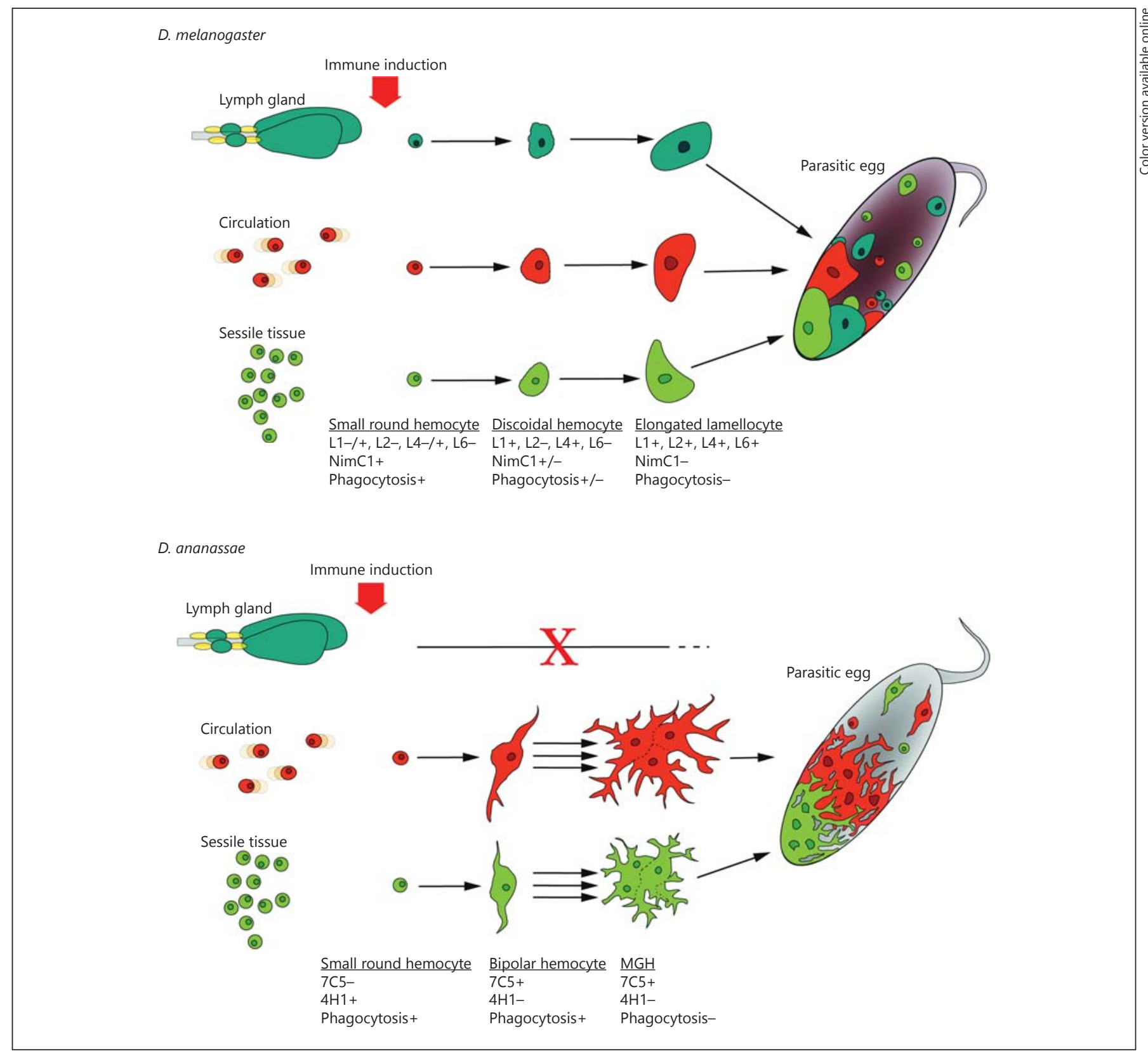

Fig. 7. Models of antiparasitic immune responses of D. melanogaster and $D$. ananassae. Small round phagocytic hemocytes, plasmatocytes, serve as precursors of the encapsulating blood cells, lamellocytes of $D$. melanogaster and MGHs of $D$. ananassae. The functional transition of phagocytic hemocytes to encapsulating cells is achieved through intermediate hemocyte types and is ac- companied by characteristic changes in antigen expression patterns (indicated under each cell differentiation stage). Although the capsules formed by MGHs in D. ananassae and by lamellocytes in $D$. melanogaster are structurally similar, in D. ananassae melanization is not involved in the elimination of the parasite.

melanogaster - parasitoid eggs are eliminated through a localized oxidative stress response in the absence of melanization [30]. The infection of larvae by generalist parasitoids proved more successful than in the case of L. boulardi, a specialist wasp of the melanogaster subgroup. 
Phenomena similar to this were also observed in D. melanogaster [8], where the directed destruction of capsuleforming cells was seen, which correlated with the success of parasitosis. Our observations are in agreement with previous findings and demonstrate the importance of MGHs as capsule-forming immune cells.

The results on the compartmental origin of MGHs yield important insight into the function of the immune compartments. The macromorphology of the hematopoietic compartments of $D$. ananassae is similar to that of their D. melanogaster counterparts; however, they appear to be functionally more specialized. In $D$. ananassae, the marker analysis did not reveal functional zones within the primary lymph gland lobes. The overlapping expression of Hml-GFP and Pxn-GFP and the presence of the Collier protein resemble the accessory lobes of the $D$. melanogaster lymph gland, which do not take part in the larval immune response. In D. melanogaster, the concerted action of the first lymph gland lobes, the sessile tissue and circulating hemocytes is observed in the defense against the parasite $[3,10,17]$. In contrast, the lymph gland in $D$. ananassae is not involved in the differentiation and release of hemocytes in response to parasitoid infection. This therefore suggests that the main role of this compartment is to provide plasmatocytes in the pupa and in the adult fly.

The development of MGHs is to some extent indicative of the development of encapsulating cells in general. Similarly to encapsulating lamellocytes of $D$. melanogaster [10,23], MGHs develop from phagocytic cells through the transition of an intermediate filariform cell type, which has the characteristics of both phagocytic and encapsulating cells. Similarly to the intermediate, discoidal cells found in D. melanogaster lamellocyte differentiation [10], these filariform cells engulf microbes and express markers characteristic of MGHs. MGHs are formed by the fusion of several mono- and multinucleated cells. As the fusion occurs exclusively between immune-induced hemocytes, a tight recognition process must be involved in the initiation of the fusion.

Recent studies revealed morphological and functional variations among capsule-forming cells of different dipteran species. Havard et al. [31] described the pseudopodocyte in D. affinis and D. obscura. Pseudopodocytes are capable of phagocytosis and share several common traits with plasmatocytes, and they are also involved in capsule formation. In the drosophilid Zaprionus indianus, both filariform cells, nematocytes and lamellocytes, are present in response to parasitosis and eliminate the parasite in the absence of melanization [32], similarly as

in D. ananassae. Developmental and cell differentiation studies in different Drosophila species are often hindered by the lack of transgenic tools and the observed genomic instability [33]. However, our results show that transgenic systems can be maintained and used to study hemocyte differentiation in D. ananassae.

In mammals, multinucleated giant cells have been known for decades [19]. They are formed after the fusion of macrophage-monocyte lineage-derived blood cells early after infection with certain pathogens and large foreign bodies [34, 35]. As MGHs of Drosophila are motile cells with filamentous projections, derived from phagocytic cells and formed by cell fusion, they strongly resemble giant cells found in mammalian foreign body-type granulomas. Since the mechanism of fusion, the signaling pathways regulating their formation and their biological importance are only partially understood, we anticipate that this surprising similarity may allow the utilization of D. ananassae as a model for a better understanding of the development, structure and function of multinucleated giant blood cells and granuloma formation in general.

\section{Acknowledgments}

We appreciate the helpful comments of the unnamed referees. We are grateful Olga Kovalcsik, Anita Balázs, Szilvia Tápai and Anikó Képíró for their technical assistance. We thank Prof. Bashisth Narayan Singh (Banaras University, Delhi) for his help in the initial part of the project, Prof. Todd Schlenke for L. heterotoma and L. victoriae, and Dr. Michèle Crozatier for the anti-Collier antibody. The research was supported by grants from the Hungarian Science Foundation (OTKA grant NK 101730) and by the European Union, and cofinanced by the European Social Fund, TÁMOP 4.2.2.A-11/1/KONV-2012-0035 (I.A.), and by the European Union and the State of Hungary, cofinanced by the European Social Fund in the framework of TÁMOP 4.2.4.A/2-11-1-2012-0001 'National Excellence Program' (V.H.).

References

Innate Immun 2015;7:340-353 DOI: $10.1159 / 000369618$ $\checkmark 1$ Adams DO: The granulomatous inflammatory response. A review. Am J Pathol 1976;84: 164-192.

2 Rottino A, Kopac MJ: Pathogenesis of spontaneously occurring and induced melanotic granuloma in Drosophila melanogaster. Prog Exp Tumor Res 1966;8:66-94.

-3 Márkus R, Laurinyecz B, Kurucz E, Honti V, Bajusz I, Sipos B, et al: Sessile hemocytes as a hematopoietic compartment in Drosophila melanogaster. Proc Natl Acad Sci U S A 2009; 106:4805-4809.

4 Keebaugh ES, Schlenke TA: Adaptive evolution of a novel Drosophila lectin induced by parasitic wasp attack. Mol Biol Evol 2012;29: 565-577. 
5 Mortimer NT, Kacsoh BZ, Keebaugh ES, Schlenke TA: Mgat1-dependent N-glycosylation of membrane components primes Drosophila melanogaster blood cells for the cellular encapsulation response. PLoS Pathog 2012;8:e1002819.

6 Nappi AJ: Hemocytic changes associated with the encapsulation and melanization of some insect parasites. Exp Parasitol 1973;33:285302.

7 Carton Y, Boulétreau M: Encapsulation ability of Drosophila melanogaster: a genetic analysis. Dev Comp Immunol 1985;9:211219.

8 Rizki TM, Rizki RM, Carton Y: Leptopilina heterotoma and L. boulardi: strategies to avoid cellular defense responses of Drosophila melanogaster. Exp Parasitol 1990;70:466475.

9 Rizki TM, Rizki RM: Properties of the larval hemocytes of Drosophila melanogaster. Experientia 1980;36:1223-1226.

- 10 Honti V, Csordás G, Márkus R, Kurucz E, Jankovics F, Andó I: Cell lineage tracing reveals the plasticity of the hemocyte lineages and of the hematopoietic compartments in Drosophila melanogaster. Mol Immunol 2010;47:1997-2004.

-11 Rizki TM, Rizki RM: Functional significance of the crystal cells in the larva of Drosophila melanogaster. J Biophys Biochem Cytol 1959; 5:235-240.

12 Sorrentino RP, Carton Y, Govind S: Cellular immune response to parasite infection in the Drosophila lymph gland is developmentally regulated. Dev Biol 2002;243:65-80.

-13 Krzemień J, Dubois L, Makki R, Meister M, Vincent A, Crozatier M: Control of blood cell homeostasis in Drosophila larvae by the posterior signalling centre. Nature 2007;446: 325-328.

14 Krzemień J, Oyallon J, Crozatier M, Vincent A: Hematopoietic progenitors and hemocyte lineages in the Drosophila lymph gland. Dev Biol 2010;346:310-319.

-15 Mandal L, Martinez-Agosto JA, Evans CJ, Hartenstein V, Banerjee U: A Hedgehog- and Antennapedia-dependent niche maintains
Drosophila haematopoietic precursors. Nature 2007;446:320-324.

16 Zettervall C-J, Anderl I, Williams MJ, Palmer R, Kurucz E, Ando I, et al: A directed screen for genes involved in Drosophila blood cell activation. Proc Natl Acad Sci U S A 2004;101: 14192-14197.

17 Avet-Rochex A, Boyer K, Polesello C, Gobert $\mathrm{V}$, Osman D, Roch F, et al: An in vivo RNA interference screen identifies gene networks controlling Drosophila melanogaster blood cell homeostasis. BMC Dev Biol 2010;10:65.

18 Russo J, Dupas S, Frey F, Carton Y, Brehelin M: Insect immunity: early events in the encapsulation process of parasitoid (Leptopilina boulardi) eggs in resistant and susceptible strains of Drosophila. Parasitology 1996;112: 135-142.

19 Papadimitriou JM, Sforsina D, Papaelias L: Kinetics of multinucleate giant cell formation and their modification by various agents in foreign body reactions. Am J Pathol 1973;73: 349-364.

20 Kurucz E, Váczi B, Márkus R, Laurinyecz B, Vilmos P, Zsámboki J, et al: Definition of Drosophila hemocyte subsets by cell-type specific antigens. Acta Biol Hung 2007; 58(suppl):95-111.

21 Köhler G, Milstein C: Continuous cultures of fused cells secreting antibody of predefined specificity. Nature 1975;256:495-497.

22 Csordás G, Varga GIB, Honti V, Jankovics F, Kurucz É, Andó I: In vivo immunostaining of hemocyte compartments in Drosophila for live imaging. PLoS One 2014;9:e98191.

23 Stofanko M, Kwon SY, Badenhorst P: Lineage tracing of lamellocytes demonstrates Drosophila macrophage plasticity. PLoS One 2010;5:e14051.

24 Goto A, Kadowaki T, Kitagawa Y: Drosophila hemolectin gene is expressed in embryonic and larval hemocytes and its knock down causes bleeding defects. Dev Biol 2003;264: 582-591.

25 Stramer B, Wood W, Galko MJ, Redd MJ, Jacinto A, Parkhurst SM, et al: Live imaging of wound inflammation in Drosophila embryos reveals key roles for small GTPases during in vivo cell migration. J Cell Biol 2005;168:567573.

26 Chiu H, Sorrentino RP, Govind S: Suppression of the Drosophila cellular immune response by Ganaspis xanthopoda. Adv Exp Med Biol 2001;484:161-167.

27 De Jong R, Kaiser L: Odor learning by Leptopilina boulardi, a specialist parasitoid (Hymenoptera: Eucoilidae). J Insect Behav 1991;4: 743-750.

28 Schlenke TA, Morales J, Govind S, Clark AG: Contrasting infection strategies in generalist and specialist wasp parasitoids of Drosophila melanogaster. PLoS Pathog 2007;3:e158.

29 Morales J, Chiu H, Oo T, Plaza R, Hoskins S, Govind S: Biogenesis, structure, and immune-suppressive effects of virus-like particles of a Drosophila parasitoid, Leptopilina victoriae. J Insect Physiol 2005;51:181-195.

30 Nappi AJ, Vass E, Frey F, Carton Y: Superoxide anion generation in Drosophila during melanotic encapsulation of parasites. Eur J Cell Biol 1995;68:450-456.

31 Havard S, Doury G, Ravallec M, Brehélin M, Prévost G, Eslin P: Structural and functional characterization of pseudopodocyte, a shaggy immune cell produced by two Drosophila species of the obscura group. Dev Comp Immunol 2012;36:323-331.

32 Kacsoh BZ, Bozler J, Schlenke TA: A role for nematocytes in the cellular immune response of the drosophilid Zaprionus indianus. Parasitology 2014;141:697-715.

-33 Schaeffer SW, Bhutkar A, McAllister BF, Matsuda M, Matzkin LM, O'Grady PM, et al: Polytene chromosomal maps of 11 Drosophila species: the order of genomic scaffolds inferred from genetic and physical maps. Genetics 2008; 179:1601-1655.

34 Chambers TJ: Fusion of macrophages following simultaneous attempted phagocytosis of glutaraldehyde-fixed red cells. J Pathol 1977; 122:71-80.

- 35 Thiede A, Sonntag HG, Leder LD, MüllerRuchholtz W: Partially common antigenicity of macrophages, epitheloid cells and foreign body giant cells. Experimental studies on rats (in German). Exp Pathol (Jena) 1977;14:16-23. 